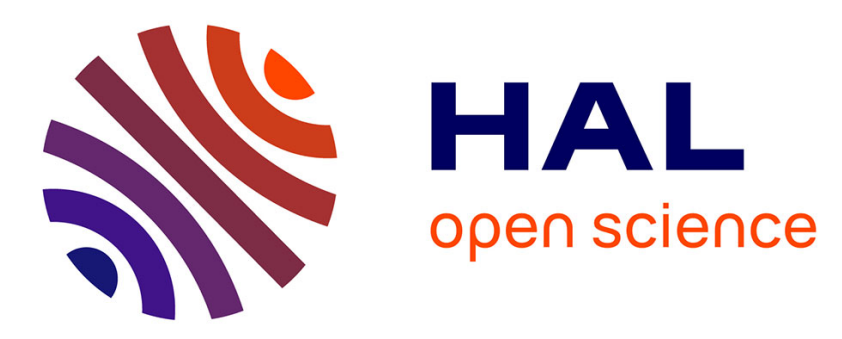

\title{
Imputation multiple et répartition des données manquantes dans les cohortes: exemple de la fécondation in vitro
}

Noémie Soullier, Elise de La Rochebrochard, Jean Bouyer

\section{To cite this version:}

Noémie Soullier, Elise de La Rochebrochard, Jean Bouyer. Imputation multiple et répartition des données manquantes dans les cohortes: exemple de la fécondation in vitro. Epidemiology and Public Health = Revue d'Epidémiologie et de Santé Publique, 2008, 56 (Suppl. 5), pp.S276. 10.1016/j.respe.2008.06.077 . hal-02423106

\section{HAL Id: hal-02423106 https://hal.science/hal-02423106}

Submitted on 18 Feb 2020

HAL is a multi-disciplinary open access archive for the deposit and dissemination of scientific research documents, whether they are published or not. The documents may come from teaching and research institutions in France or abroad, or from public or private research centers.
L'archive ouverte pluridisciplinaire HAL, est destinée au dépôt et à la diffusion de documents scientifiques de niveau recherche, publiés ou non, émanant des établissements d'enseignement et de recherche français ou étrangers, des laboratoires publics ou privés. 
Soullier Noémie, La Rochebrochard Elise (de), Bouyer Jean, 2008, « Imputation multiple et répartition des données manquantes dans les cohortes : exemple de la fécondation in vitro », Revue d’Epidémiologie et de Santé Publique, 56(Suppl. 5), S276. DOI: 10.1016/j.respe.2008.06.077.

\title{
Imputation multiple et répartition des données manquantes dans les cohortes : exemple de la fécondation in vitro
}

\author{
Noémie Soullier ${ }^{a, b, c}$, Elise de la Rochebrochard ${ }^{a, b, c}$, Jean Bouyer ${ }^{a, b, c}$ \\ a Inserm unité 822, Le Kremlin-Bicêtre, France \\ b Ined, Le Kremlin-Bicêtre, France \\ c Faculté de médecine Paris-Sud, hôpital de Bicêtre, université Paris-Sud 11, Le Kremlin-Bicêtre, France
}

Objectif. L'attrition dans les cohortes a des conséquences importantes en termes de biais de sélection et de puissance. Pour traiter ces données manquantes, des méthodes telles que l’imputation multiple ont été développées. Son principe est de remplacer chaque valeur manquante par plusieurs valeurs possibles, puis de combiner les estimations obtenues en une estimation unique. En fécondation in vitro (FIV), l'attrition correspond à des arrêts de traitement sans avoir eu d'enfant. Notre objectif est de tester différentes stratégies d’imputation multiple selon le schéma d'attrition, c'est-à-dire selon la répartition des interruptions de traitement entre les tentatives.

Méthode. Une population de 100000 individus a été simulée, afin de connaître le « taux vrai » de la population. Les interruptions de traitement ont ensuite été réparties au cours des tentatives. Différentes répartitions des interruptions ont été créées, représentant une attrition stable, décroissante ou croissante au cours du temps. Des cohortes de 2000 individus y ont été tirées au sort et répliquées 500 fois. Le taux cumulé de succès en FIV a été estimé par imputation multiple, avec différentes répartitions du nombre d'imputations entre les tentatives. Pour un même nombre 
d'imputations finales, des répartitions croissantes, monotones et décroissantes des nombres d'imputation à chaque tentative ont été mises en place. Le biais et l'écart-type de l'estimateur, ainsi que le taux de recouvrement de son intervalle de confiance, ont été calculés.

Résultats. Si la répartition du nombre d'imputations ne semble pas primordiale, il semble nécessaire d'effectuer les imputations sur chaque temps de mesure. Le schéma d'attrition ne semble pas avoir d'effet majeur sur les performances de la méthode.

Conclusion. Cette étude pourra profiter aux cohortes confrontées à un problème d'attrition, dans le cadre de l'estimation de l'occurrence d'un événement, où les perdus de vue seraient traités comme des données manquantes. 